Revista Verde de Agroecologia e Desenvolvimento Sustentável

http://www.gvaa.com.br/revista/index.php/RVADS

ARTIGO CIENTÍFICO

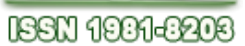

DOI: http://dx.doi.org/10.18378/rvads.v10i3.2716

\title{
Análise da adequação de eventos de precipitação extrema na Amazônia Ocidental em modelos estáticos: Rondônia
}

\section{Analysis of fitness for extreme rainfall events in western amazon in static models: state Rondônia}

\author{
Vinicius Alexandre Sikora de Souza ${ }^{1}$, Marcos Leando Alves Nunes ${ }^{2}$, Sandra Ferronatto Francener ${ }^{3}$, Ana Lúcia Denardin da \\ $\operatorname{Rosa}^{4}$
}

RESUMO: Este estudo objetivou estimar a função Intensidade-Duração-Frequência (IDF) de eventos pluviométricos extremos a partir dos dados de precipitação das estações pluviométricas instaladas no estado de Rondônia, de modo que posteriormente tais informações possam ser utilizadas no dimensionamento de obras hidráulicas. Utilizou-se 41 estações pluviométricas com séries históricas acima de 10 anos, disponibilizadas pela Agência Nacional de Águas (ANA). Essas séries passaram inicialmente pelo teste de aderência Kolmogorov-Smirnov (KS), a fim de verificar o ajuste das mesmas as distribuições: Normal, Log-Normal, Exponencial, Gama, Gumbel, Weibull e Logística. O trabalho denotou que o teste de aderência Kolmogorov-Smirnov de forma geral forneceu uma expressiva aceitação na maioria das distribuições estatística testadas.

Palavras-chave: chuvas intensas, modelagem, hidroestatística

\begin{abstract}
This study aimed to estimate the Intensity - Duration - Frequency (IDF) function extreme rainfall events from the data of precipitation of rainfall stations located in the State of Rondônia, so that such information can be later used in hydraulic structures. We used 41 rainfall stations with historical series over 10 years, provided by the National Water Agency (ANA). These series originally started by adherence Kolmogorov -Smirnov (KS) in order to check the fit of the same distributions: Normal, Log- Normal, Exponential, Gamma, Gumbel, Weibull and Logistics. Work denoted that the Kolmogorov - Smirnov test of adherence generally provided a significant acceptance in most of the tested statistical distributions.
\end{abstract}

Keywords: intense rains, modeling, hidroestatística

\footnotetext{
*Autor para correspondência

Recebido para publicação em 11/06/2014; aprovado em 10/07/2015

${ }^{1}$ Mestrando em Engenharia Civil pela Universidade Federal do Rio de Janeiro (UFRJ); Especialista em Engenharia e Segurança do Trabalho pela FASA;

Engenheiro Ambiental pela Universidade Federal de Rondônia. E-mail: vass1000@ hotmail.com

${ }^{2}$ Mestre em Engenharia Civil pela Universidade Federal do Rio de Janeiro (UFRJ) e Engenheiro Ambiental pela Universidade Federal de Rondônia. E-mail: marcosbatarelli@hotmail.com

${ }^{3}$ Engenheira Ambiental pela Universidade Federal de Rondônia - UNIR. E-mail: sandraffrancener@hotmail.com

${ }^{4}$ Professora assistente da Fundação Universidade Federal de Rondônia e Mestre em Engenharia Civil pela Universidade Federal de Santa Maria. E-mail:

eng.analucia@yahoo.com.br
} 


\section{INTRODUÇÃO}

O Brasil apresentou, principalmente após a década de 60, um crescimento significativo da população urbana. A ocorrência deste fenômeno nas últimas décadas transformou o Brasil num país essencialmente urbano ( $83 \%$ de população urbana), gerando uma população urbana com uma infraestrutura inadequada (TUCCI, 1999; 2008).

Os efeitos desse processo ocorrido de forma desorganizada se propagam por todo o aparelhamento urbano relativo aos recursos hídricos. Principalmente ao que concerne ao planejamento e construção de obras hidráulicas e hidrológicas, para a minimização de impactos sócioambientais como as enchentes, pelo planejamento da drenagem urbana (OLIVEIRA et al., 2011; TUCCI, 2007).

O conhecimento de eventos hidrológicos extremos é um requisito em projetos de drenagem, impermeabilização e outras obras de engenharia, seja em áreas urbanas ou rurais, isso porque, permite que o projetista considere os riscos existentes com a execução da obra e associe à melhor alternativa, do ponto de vista econômico, sem se desvencilhar das questões técnicas de desempenho e segurança. Todavia, tais dados são incipientes e restritos em algumas localidades.

A precipitação pluviométrica, dentre os elementos hidrológicos, é o que mais interfere na vida humana, uma vez que, se configura como a principal entrada de água no sistema hidrológico, tornando outras variantes como a vazão e a infiltração, intimamente ligadas a sua ocorrência. Em virtude de sua larga influência sob áreas povoadas, positivas ou não, a chuva pode ser considera a principal forma de suprimento hídrico para as atividades humanas e econômicas (ALMEIDA et al., 2011).

Dessa forma, torna-se de grande importância o conhecimento e a previsão das características das precipitações pluviométricas, destacando a elucidação de sua intensidade máxima, duração desse fenômeno e o período que tal evento possa voltar a ocorrer.

Nesse sentindo, uma forma amplamente empregada para a caracterização das chuvas extremas em uma determina localidade é a utilização de curvas de intensidade-duraçãofrequência (IDF). Essas consistem em modelos matemáticos semi-empíricos que prevêem a intensidade precipitada por meio da duração e distribuição temporal. Destaca-se que a inferência da pluviosidade extrema é possível devido a tais eventos se ajustarem a distribuições probabilísticas, propiciando que sejam modeladas estatisticamente.

Dentre modelos de distribuições teóricas, os que mais se adequam de a modelagem segundo Souza (2012) são as distribuições: Normal, Log-Normal, Exponencial, Gama, Gumbel, Weibull e Logística.

No Brasil, no entanto estes fenômenos ainda são pouco estudados principalmente em regiões como o estado de Rondônia, localizado na Amazônia Ocidental. Esse estado é praticamente desprovido de informações de chuvas intensas. Assim, a realização de projetos de obras hidráulicas são efetuados com informações de postos meteorológicos próximos ou de características climatológicas similares a da localidade na qual o projeto é realizado.

Tal procedimento, entretanto, pode levar a estimativas pouco confiáveis, devido a essa prática apresentar o risco das obras serem subestimadas, trazendo problemas, principalmente, de ordem social, assim como, serem hiperestimadas, onerando os custos das mesmas.

Em vista ao exposto, este estudo objetivou verificar dentre sete distribuições estatísticas mencionadas por Souza (2012), a que melhor se ajusta a distribuição das chuvas extremas do estado de Rondônia, para que posteriormente tal informação possa ser utilizada na tomada de decisão e no estudo desses fenômenos.

\section{MATERIAL E MÉTODOS}

\section{ÁREA DE ESTUDO}

O Estado de Rondônia (FIGURA 1) localiza-se na Amazônia Ocidental entre os paralelos de $7^{\circ} 58^{\prime}$ e $13^{\circ} 43^{\prime}$ de Latitude Sul e meridianos de $59^{\circ} 50^{\prime}$ e $66^{\circ} 48^{\prime}$ de Longitude Oeste. Rondônia é composto por 52 municípios, sendo o mais populoso a capital do estado, Porto Velho, que possui 428.527 habitantes (IBGE, 2010).

$\mathrm{O}$ estado de Rondônia apresenta altitudes que variam entre 70 e 600 metros, sendo suas principais unidades de relevo: Planícies Amazônicas; Depressões (Amazônia Meridional, Guaporé e Solimões); Planaltos (Amazônia Meridional e dos Parecis); Pantanal do Guaporé. Os tipos de vegetação encontrados na região são as Florestas Ombrófila Aberta, Florestas Ombrófilas Densas (Floresta Amazônica), Florestas Estacionais Semidecidual, Savanas e Vegetação Aluvial, com predominância de Florestas Densas a noroeste do estado (BRASIL, 1978).

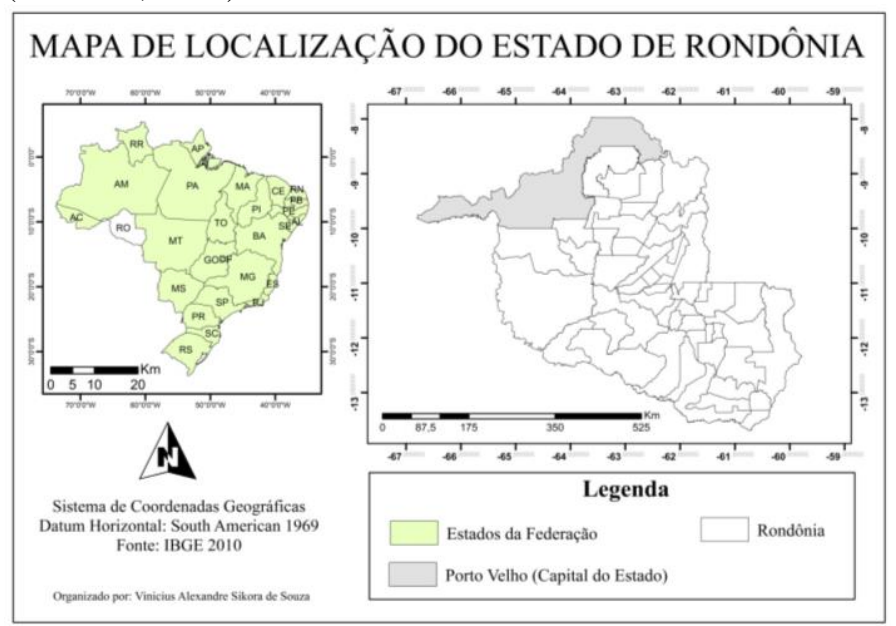

Figura 1. Localização do estado de Rondônia

\section{Clima}

$\mathrm{Na}$ classificação de Köppen, o clima do estado de Rondônia é caracterizado como AW (tropical-quente e úmido), com média climatológica da temperatura do ar durante o mês mais frio superior a $18^{\circ} \mathrm{C}$ (megatérmico) e um período seco bem definido durante a estação de inverno, quando ocorre no Estado um moderado déficit hídrico com índices pluviométricos inferiores a $50 \mathrm{~mm} / \mathrm{mês}$. (RONDÔNIA, 2009).

A chuva em Rondônia é de monções e, portanto, só ocorre durante a época do ano em que o continente é aquecido acima dos níveis de temperatura da superfície do oceano e se torna um centro de convecção, onde há uma ampla oferta de umidade para a condensação (BUTT et al., 2011). 
A precipitação pluviométrica anual na região central do estado é, em média, acima de $2.000 \mathrm{~mm}$, com umidade relativa do ar média de $82 \%$ e temperatura média anual oscilando de $24^{\circ} \mathrm{C}$ na estação chuvosa a $25^{\circ} \mathrm{C}$ na seca (AGUIAR et al., 2006; WEBLER et al., 2007).

Esta região, segundo a Secretária do Estado de Desenvolvimento Ambiental - SEDAM - (2009), não sofre grandes influências da continentalidade, ou seja, maior ou menor distância em relação ao mar.

\section{Análise dos dados}

\section{Aquisição dos dados}

Os dados de precipitação utilizados neste estudo foram obtidos por meio do banco de dados disponibilizados pela
Agência Nacional de Águas (ANA). Destaca-se que as informações desse banco vêm sendo utilizadas em vários estudos similares, dentre os quais: Arantes et al. (2009); Butt et al. (2011); Fietz \& Comunell (2006) e Oliveira et al. (2010).

Esse órgão mantém em seus registros dados de precipitação pluviométrica acumulados em períodos de um dia para 93 estações pluviométricas do estado de Rondônia, no entanto, apenas 41 apresentaram um conjunto de dados com uma série temporal igual ou superior a 10 anos, sendo tal característica explicitada por Fietz \& Comunell (2006) como requisito para a modelagem de eventos extremos. Desta forma, somente os dados dessas 41 estações foram utilizados.

$\mathrm{Na}$ Tabela 1 apresentam-se algumas informações mais detalhadas das estações utilizadas no estudo e, na Figura 2 nota-se a localização das mesmas.

Tabela 1. Dados das estações pluviométricas amostradas

\begin{tabular}{|c|c|c|c|c|c|c|}
\hline \multirow{2}{*}{ Estação } & \multirow{2}{*}{ Código } & \multirow{2}{*}{ Série (anos) } & \multirow{2}{*}{$\begin{array}{c}\text { Meses } \\
\text { efetivos }\end{array}$} & \multirow{2}{*}{ Município } & \multicolumn{2}{|c|}{ Localização } \\
\hline & & & & & Latitude & Longitude \\
\hline 1 & 1360002 & $1983-2010$ & 333 & $\begin{array}{l}\text { Pimenteiras do } \\
\text { Oeste }\end{array}$ & $13^{\circ} 28^{\prime} 47^{\prime \prime} \mathrm{S}$ & $61^{\circ} 02^{\prime} 47^{\prime \prime} \mathrm{O}$ \\
\hline 2 & 1360001 & $1983-2010$ & 336 & Cerejeiras & $13^{\circ} 11^{\prime} 48^{\prime \prime} \mathrm{S}$ & $60^{\circ} 49^{\prime} 24^{\prime \prime} \mathrm{O}$ \\
\hline 3 & 1360000 & $1983-2010$ & 334 & $\begin{array}{l}\text { Colorado do } \\
\text { Oeste }\end{array}$ & $13^{\circ} 06^{\prime} 51^{\prime \prime} \mathrm{S}$ & $60^{\circ} 32^{\prime} 54^{\prime \prime} \mathrm{O}$ \\
\hline 4 & 1264000 & $1983-2010$ & 329 & Costa Marques & $12^{\circ} 25^{\prime} 37^{\prime \prime} \mathrm{S}$ & $64^{\circ} 25^{\prime} 21^{\prime \prime} \mathrm{O}$ \\
\hline 5 & 1262001 & $1999-2010$ & 139 & $\begin{array}{c}\text { Alta Floresta do } \\
\text { Oeste }\end{array}$ & $12^{\circ} 36^{\prime} 05^{\prime \prime} \mathrm{S}$ & $62^{\circ} 10^{\prime} 42^{\prime \prime} \mathrm{O}$ \\
\hline 6 & 1262000 & $1980-2010$ & 352 & Costa Marques & $12^{\circ} 51^{\prime} 05^{\prime \prime} \mathrm{S}$ & $62^{\circ} 53^{\prime} 57^{\prime \prime} \mathrm{O}$ \\
\hline 7 & 1261001 & $1999-2010$ & 130 & Parecis & $12^{\circ} 12^{\prime} 33^{\prime \prime} \mathrm{S}$ & $61^{\circ} 37^{\prime} 43^{\prime \prime} \mathrm{O}$ \\
\hline 8 & 1261000 & $1983-2006$ & 260 & Chunpinguia & $12^{\circ} 29^{\prime} 16^{\prime \prime} \mathrm{S}$ & $61^{\circ} 02^{\prime} 47^{\prime \prime} \mathrm{O}$ \\
\hline 9 & 1260001 & $1967-2003$ & 337 & Vilhena & $12^{\circ} 42^{\prime} 00^{\prime \prime} \mathrm{S}$ & $60^{\circ} 05^{\prime} 00^{\prime \prime} \mathrm{O}$ \\
\hline 10 & 1164001 & $1983-2002$ & 229 & Guajará-Mirim & $11^{\circ} 22^{\prime} 00^{\prime \prime} \mathrm{S}$ & $64^{\circ} 53^{\prime} 11^{\prime \prime} \mathrm{O}$ \\
\hline 11 & 1164000 & $1976-1989$ & 167 & Guajará-Mirim & $11^{\circ} 04^{\prime} 00^{\prime \prime} \mathrm{S}$ & $64^{\circ} 05^{\prime} 00^{\prime \prime} \mathrm{O}$ \\
\hline 12 & 1161003 & $1999-2010$ & 131 & $\begin{array}{c}\text { Ministro } \\
\text { Andreazza }\end{array}$ & $11^{\circ} 11^{\prime} 49^{\prime \prime} \mathrm{S}$ & $61^{\circ} 31^{\prime} 41^{\prime \prime} \mathrm{O}$ \\
\hline 13 & 1161002 & $1983-2010$ & 315 & Rolim de Moura & $11^{\circ} 44^{\prime} 59^{\prime \prime} \mathrm{S}$ & $61^{\circ} 46^{\prime \prime} 35^{\prime} \mathrm{O}$ \\
\hline 14 & 1161001 & $1980-2010$ & 372 & Pimenta Bueno & $11^{\circ} 41^{\prime} 01^{\prime \prime} \mathrm{S}$ & $61^{\circ} 11^{\prime} 32^{\prime \prime} \mathrm{O}$ \\
\hline 15 & 1161000 & $1977-2010$ & 395 & Cacoal & $11^{\circ} 26^{\prime} 27^{\prime \prime} \mathrm{S}$ & $61^{\circ} 29^{\prime} 02^{\prime \prime} \mathrm{O}$ \\
\hline 16 & 1160002 & $1982-2010$ & 334 & Pimenta Bueno & $11^{\circ} 44^{\prime} 56^{\prime \prime} \mathrm{S}$ & $60^{\circ} 52^{\prime} 04^{\prime \prime} \mathrm{O}$ \\
\hline 17 & 1160000 & $1977-2010$ & 393 & Pimenta Bueno & $12^{\circ} 00^{\prime} 55^{\prime \prime} \mathrm{S}$ & $60^{\circ} 51^{\prime} 18^{\prime \prime} \mathrm{O}$ \\
\hline 18 & 1065002 & $1972-2010$ & 451 & Guajará-Mirim & $10^{\circ} 47^{\prime} 33^{\prime \prime} \mathrm{S}$ & $65^{\circ} 20^{\prime} 52^{\prime \prime} \mathrm{O}$ \\
\hline 19 & 1063001 & $1980-2010$ & 354 & Ariquemes & $09^{\circ} 45^{\prime} 39^{\prime \prime} \mathrm{S}$ & $63^{\circ} 17^{\prime} 15^{\prime \prime} \mathrm{O}$ \\
\hline 20 & 1063000 & $1978-2010$ & 358 & Ariquemes & $10^{\circ} 30^{\prime} 18^{\prime \prime} \mathrm{S}$ & $63^{\circ} 38^{\prime} 46^{\prime \prime} \mathrm{O}$ \\
\hline 21 & 1062004 & $1986-2010$ & 285 & Theobroma & $10^{\circ} 14^{\prime} 11^{\prime \prime} \mathrm{S}$ & $62^{\circ} 20^{\prime} 45^{\prime \prime} \mathrm{O}$ \\
\hline 22 & 1062003 & $1983-2010$ & 324 & $\begin{array}{c}\text { Mirante da } \\
\text { Serra }\end{array}$ & $11^{\circ} 00^{\prime} 13^{\prime \prime} \mathrm{S}$ & $62^{\circ} 39^{\prime} 22^{\prime \prime} \mathrm{O}$ \\
\hline 23 & 1062002 & $1978-210$ & 383 & Jaru & $10^{\circ} 14^{\prime} 11^{\prime \prime} \mathrm{S}$ & $62^{\circ} 37^{\prime} 38^{\prime \prime} \mathrm{O}$ \\
\hline 24 & 1062001 & $1977-2010$ & 399 & Jaru & $10^{\circ} 26^{\prime} 45^{\prime \prime} \mathrm{S}$ & $62^{\circ} 27^{\prime} 56^{\prime \prime} \mathrm{O}$ \\
\hline 25 & 1061003 & $1987-2010$ & 285 & $\begin{array}{l}\text { Ouro Preto do } \\
\text { Oeste }\end{array}$ & $10^{\circ} 31^{\prime} 01^{\prime \prime} \mathrm{S}$ & $62^{\circ} 00^{\prime} 05^{\prime \prime} \mathrm{O}$ \\
\hline 26 & 1061001 & $1975-1997$ & 262 & Ji-Paraná & $10^{\circ} 50^{\prime} 58^{\prime \prime} \mathrm{S}$ & $61^{\circ} 55^{\prime} 50^{\prime \prime} \mathrm{O}$ \\
\hline 27 & 966001 & $1982-2010$ & 316 & Porto velho & $09^{\circ} 41^{\prime} 25^{\prime \prime} \mathrm{S}$ & $65^{\circ} 59^{\prime} 35^{\prime \prime} \mathrm{O}$ \\
\hline 28 & 966000 & $1977-2010$ & 404 & Porto velho & $09^{\circ} 45^{\prime} 20^{\prime \prime} \mathrm{S}$ & $66^{\circ} 36^{\prime} 42^{\prime \prime} \mathrm{O}$ \\
\hline 29 & 965001 & $1976-2010$ & 377 & Porto velho & $09^{\circ} 42^{\prime} 11^{\prime \prime} \mathrm{S}$ & $65^{\circ} 21^{\prime} 53^{\prime \prime} \mathrm{O}$ \\
\hline 30 & 964001 & $1978-2002$ & 290 & Porto velho & $09^{\circ} 30^{\prime} 59^{\prime \prime} \mathrm{S}$ & $64^{\circ} 48^{\prime} 44^{\prime \prime} \mathrm{O}$ \\
\hline
\end{tabular}


(Continuação) Tabela 1. Dados das estações pluviométricas amostradas

\begin{tabular}{|c|c|c|c|c|c|c|}
\hline \multirow{2}{*}{ Estação } & \multirow{2}{*}{ Código } & \multirow{2}{*}{ Série (anos) } & \multirow{2}{*}{$\begin{array}{c}\text { Meses } \\
\text { efetivos }\end{array}$} & \multirow{2}{*}{ Município } & \multicolumn{2}{|c|}{ Localização } \\
\hline & & & & & Latitude & Longitude \\
\hline 31 & 963009 & $1997-2010$ & 162 & Ariquemes & $09^{\circ} 28^{\prime} 00^{\prime \prime} \mathrm{S}$ & $63^{\circ} 15^{\prime} 00^{\prime \prime} \mathrm{O}$ \\
\hline 32 & 963006 & $1983-2001$ & 223 & Ariquemes & $09^{\circ} 53^{\prime} 14^{\prime \prime} \mathrm{S}$ & $62^{\circ} 59^{\prime} 17^{\prime \prime} \mathrm{O}$ \\
\hline 33 & 963004 & $1980-2010$ & 366 & Ariquemes & $09^{\circ} 53^{\prime} 14^{\prime \prime} \mathrm{S}$ & $62^{\circ} 59^{\prime} 16^{\prime \prime} \mathrm{O}$ \\
\hline 34 & 963001 & $1977-2010$ & 387 & Porto velho & $09^{\circ} 15^{\prime} 38^{\prime \prime} \mathrm{S}$ & $63^{\circ} 09^{\prime} 43^{\prime \prime} \mathrm{O}$ \\
\hline 35 & 963000 & $1975-2010$ & 420 & Ariquemes & $09^{\circ} 55^{\prime} 54^{\prime \prime} \mathrm{S}$ & $63^{\circ} 03^{\prime} 25^{\prime \prime} \mathrm{O}$ \\
\hline 36 & 962001 & $1981-2007$ & 308 & Porto velho & $09^{\circ} 10^{\prime} 45^{\prime \prime} \mathrm{S}$ & $62^{\circ} 57^{\prime} 11^{\prime \prime} \mathrm{O}$ \\
\hline 37 & 962000 & $1978-2010$ & 378 & $\begin{array}{c}\text { Machadinho do } \\
\text { Oeste }\end{array}$ & $09^{\circ} 35^{\prime} 11^{\prime \prime} \mathrm{S}$ & $62^{\circ} 23^{\prime} 38^{\prime \prime} \mathrm{O}$ \\
\hline 38 & 961003 & $1987-2009$ & 273 & $\begin{array}{c}\text { Machadinho do } \\
\text { Oeste }\end{array}$ & $09^{\circ} 40^{\prime} 53^{\prime \prime} \mathrm{S}$ & $61^{\circ} 58^{\prime} 44^{\prime \prime} \mathrm{O}$ \\
\hline 39 & 863003 & $1975-2002$ & 297 & Candeias do Jamari & $08^{\circ} 45^{\prime} 35^{\prime \prime} \mathrm{S}$ & $63^{\circ} 27^{\prime} 45^{\prime \prime} \mathrm{O}$ \\
\hline 40 & 863000 & $1961-2007$ & 564 & Porto velho & $08^{\circ} 46^{\prime} 00^{\prime \prime} \mathrm{S}$ & $63^{\circ} 55^{\prime} 00^{\prime \prime} \mathrm{O}$ \\
\hline 41 & 862000 & $1977-2010$ & 385 & $\begin{array}{l}\text { Machadinho do } \\
\text { Oeste }\end{array}$ & $08^{\circ} 56^{\prime} 00^{\prime \prime} \mathrm{S}$ & $62^{\circ} 03^{\prime} 14^{\prime \prime} \mathrm{O}$ \\
\hline
\end{tabular}

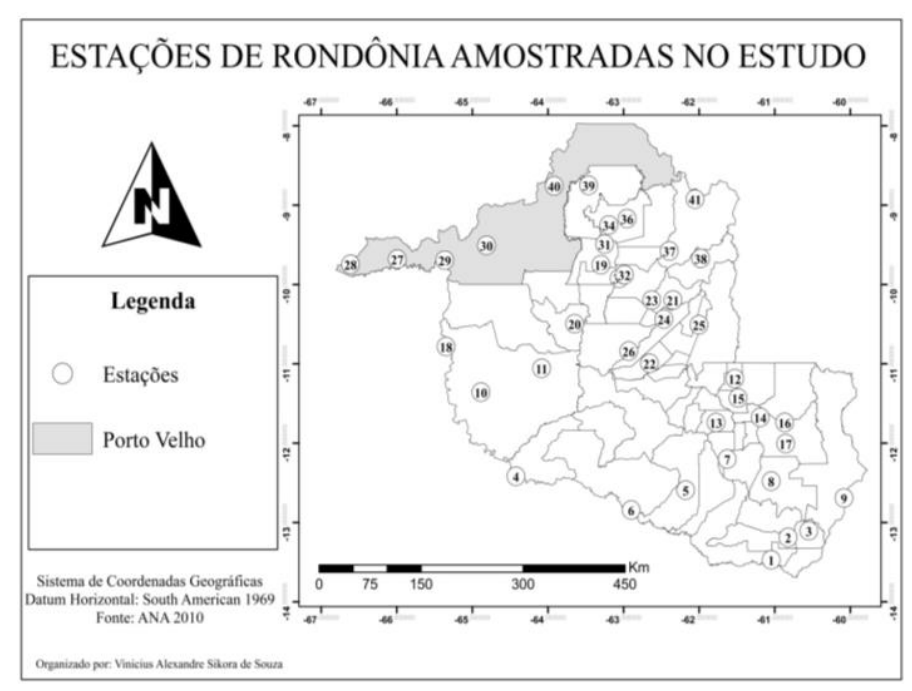

Figura 2. Localização das estações pluviométricas do estado de Rondônia utilizadas neste estudo

\section{Teste de Aderência}

$\mathrm{Na}$ verificação da distribuição estatística que melhor se ajusta aos eventos pluviométricos máximos anuais registrados nos dados coletados utilizou-se um teste de aderência.

As distribuições testadas foram as distribuições Normal, Log-Normal, Exponencial, Gama, Gumbel, Weibull e Logística.

Dentre os testes de aderência indicados por Naghettini \& Pinto (2007), optou-se pelo teste Kolmogorov-Smirnov (KS), o qual segundo os autores mencionados é um teste não paramétrico que tem como base a diferença máxima entre as funções de probabilidades acumuladas, empírica e teórica, de variáveis aleatórias contínuas, sendo o mesmo conservador quanto à magnitude do erro do tipo I.

Tal teste também é destacado por Leotti et al. (2005) como pertencente à classe suprema de estatísticas baseadas na função de distribuição empírica, pois trabalha com a maior diferença entre a distribuição empírica e a hipotética. Catalunha et al. (2002) explicitam além disso, que o KS é bastante utilizado para análise de aderências de distribuições em estudos climáticos.

Assim, o teste Kolmogorov-Smirnov para as distribuições analisadas, executado pelo software Weibull++ 7® (RELIASOFT, 2011), seguiu-se primeiramente pela conjuração da hipótese nula $\left(\mathrm{H}_{0}\right)$, que a distribuição empírica é igual a distribuição dos valores amostrais, e da hipótese alternativa $\left(\mathrm{H}_{1}\right)$, onde a assertiva da hipótese nula não seria verdadeira, ou seja, a distribuição dos dados analisados não corresponderiam a distribuição estatística analisada.

O critério de decisão foi a comparação dos parâmetros $D_{\text {calc }}$ e $D_{\text {crit }}$, onde $D_{\text {calc }}>D_{\text {crit }}$ rejeita-se a hipótese $H_{0}$ em favor de $\mathrm{H}_{1}$. Nos testes de aderências utilizaram-se um nível de significância $(\alpha)$ de 0,005 para obter um valor reduzido do erro do tipo I, ou seja, minimizar as chances para aproximadamente $100 \%$ de descartar a hipótese nula sendo a mesma verdadeira (BORGES \& FERREIRA, 2003).

\section{RESULTADOS E DISCUSSÃO}

Os resultados das avaliações de aderência estão resumidos na Tabela 2, utilizando o Teste Kolmogorov-Smirnov, a fim de verificar o ajuste dos dados das estações em relação às distribuições de probabilidade empíricas. Como explicitado anteriormente, utilizou-se o software Weibull++ 7® (RELIASOFT, 2011).

O referido programa apresenta os resultados no seguinte formato: ele faz uma relação entre os parâmetros $D_{\text {crit }}$ e $D_{\text {calc }}$ $\mathrm{e}$, as relações que se apresentam inferiores a 1 denotam que a distribuição teórica testada pode ser utilizada para prever o comportamento dos dados observados por uma determinada estação pluviométrica, pois valores em tal magnitude expressam a afirmação que o parâmetro $D_{\text {crit }}$ é superior ao parâmetro $\mathrm{D}_{\text {calc }}$, sendo este o critério de aceitação da hipótese nula $\left(\mathrm{H}_{0}\right)$.

No entanto, se tal relação apresentar-se com o valor de 1 , esse indicará que a distribuição estatística em análise não é correspondente a disposição probabilísticas dos dados de chuvas máximas, pois a afirmação de $\mathrm{D}_{\text {crit }}<\mathrm{D}_{\text {calc }}$ é verídica, o que obriga a negação da hipótese nula e aceitação da hipótese alternativa. 
Tabela 2 - Resultados dos testes de aderência utilizando KS e análise de simetria

\begin{tabular}{|c|c|c|c|c|c|c|c|c|c|}
\hline \multirow{2}{*}{ Estação } & \multicolumn{7}{|c|}{$\mathbf{D}_{\text {calc }} / \mathbf{D}_{\text {crit }}$} & \multirow{2}{*}{$\mathbf{n}$} & \multirow{2}{*}{$\begin{array}{c}\text { Coeficiente } \\
\text { de } \\
\text { Simetria }\end{array}$} \\
\hline & Normal & $\begin{array}{c}\text { Log- } \\
\text { Normal }\end{array}$ & Gama & Weibull & Logística & Exponencial & Gumbel & & \\
\hline 1 & 0,706 & 0,997 & 1,000 & 0,971 & 0,647 & 1,000 & 0,900 & 28 & 0,451 \\
\hline 2 & 0,348 & 0,028 & 1,000 & 0,213 & 0,265 & 1,000 & 0,736 & 28 & 0,608 \\
\hline 3 & 0,230 & 0,284 & 1,000 & 0,186 & 0,130 & 1,000 & 0,573 & 28 & 0,477 \\
\hline 4 & 0,254 & 0,210 & 1,000 & 0,209 & 0,397 & 1,000 & 0,676 & 28 & 0,192 \\
\hline 5 & 0,927 & 0,500 & 1,000 & 0,858 & 0,897 & 1,000 & 0,987 & 12 & $-0,242$ \\
\hline 6 & 0,483 & 0,729 & 1,000 & 0,276 & 0,574 & 1,000 & 0,116 & 31 & $-0,568$ \\
\hline 7 & 0,008 & 0,053 & 1,000 & 0,004 & 0,022 & 0,999 & 0,019 & 12 & $-0,041$ \\
\hline 8 & 0,805 & 0,696 & 1,000 & 0,872 & 0,863 & 1,000 & 0,923 & 21 & 0,642 \\
\hline 9 & 0,381 & 0,010 & 1,000 & 0,108 & 0,292 & 1,000 & 0,616 & 24 & 2,802 \\
\hline 10 & 0,010 & 0,551 & 1,000 & 0,158 & 0,002 & 0,998 & 0,128 & 19 & 0,215 \\
\hline 11 & 0,000 & 0,000 & 1,000 & 0,000 & 0,000 & 1,000 & 0,001 & 12 & 0,018 \\
\hline 12 & 0,036 & 0,024 & 1,000 & 0,072 & 0,017 & 1,000 & 0,096 & 12 & $-0,126$ \\
\hline 13 & 0,045 & 0,012 & 1,000 & 0,107 & 0,082 & 1,000 & 0,530 & 28 & 0,117 \\
\hline 14 & 0,296 & 0,081 & 1,000 & 0,557 & 0,457 & 1,000 & 0,738 & 31 & 0,575 \\
\hline 15 & 0,177 & 0,203 & 1,000 & 0,119 & 0,136 & 1,000 & 0,706 & 34 & 0,999 \\
\hline 16 & 0,384 & 0,089 & 1,000 & 0,611 & 0,482 & 1,000 & 0,826 & 29 & 2,050 \\
\hline 17 & 0,340 & 0,004 & 1,000 & 0,348 & 0,444 & 1,000 & 0,822 & 34 & 0,511 \\
\hline 18 & 0,927 & 0,500 & 1,000 & 0,858 & 0,897 & 1,000 & 0,987 & 39 & 2,015 \\
\hline 19 & 0,295 & 0,026 & 1,000 & 0,458 & 0,418 & 1,000 & 0,766 & 29 & 0,577 \\
\hline 20 & 0,996 & 0,834 & 1,000 & 0,953 & 0,996 & 1,000 & 0,999 & 31 & 3,714 \\
\hline 21 & 0,418 & 0,262 & 1,000 & 0,550 & 0,552 & 1,000 & 0,669 & 24 & 0,192 \\
\hline 22 & 0,061 & 0,449 & 1,000 & 0,020 & 0,028 & 1,000 & 0,254 & 28 & $-0,288$ \\
\hline 23 & 0,024 & 0,288 & 1,000 & 0,005 & 0,006 & 1,000 & 0,206 & 33 & 0,292 \\
\hline 24 & 0,269 & 0,009 & 1,000 & 0,192 & 0,206 & 1,000 & 0,731 & 34 & 1,892 \\
\hline 25 & 0,179 & 0,099 & 1,000 & 0,385 & 0,151 & 1,000 & 0,635 & 23 & $-0,001$ \\
\hline 26 & 0,045 & 0,000 & 1,000 & 0,046 & 0,018 & 1,000 & 0,350 & 23 & 0,760 \\
\hline 27 & 0,012 & 0,242 & 1,000 & 0,000 & 0,026 & 1,000 & 0,022 & 28 & $-0,459$ \\
\hline 28 & 0,879 & 0,640 & 1,000 & 0,847 & 0,840 & 1,000 & 0,963 & 34 & 1,518 \\
\hline 29 & 0,903 & 0,262 & 1,000 & 0,139 & 0,869 & 0,998 & 0,959 & 33 & 4,368 \\
\hline 30 & 0,415 & 0,969 & 1,000 & 0,879 & 0,424 & 0,996 & 0,176 & 24 & $-0,117$ \\
\hline 31 & 0,021 & 0,330 & 1,000 & 0,155 & 0,007 & 1,000 & 0,002 & 14 & $-0,651$ \\
\hline 32 & 0,299 & 0,708 & 1,000 & 0,388 & 0,335 & 1,000 & 0,693 & 19 & $-0,555$ \\
\hline 33 & 0,471 & 0,097 & 1,000 & 0,650 & 0,539 & 1,000 & 0,875 & 31 & 0,793 \\
\hline 34 & 0,504 & 0,140 & 1,000 & 0,455 & 0,389 & 1,000 & 0,786 & 34 & 1,113 \\
\hline 35 & 0,292 & 0,002 & 1,000 & 0,282 & 0,231 & 1,000 & 0,785 & 36 & 0,829 \\
\hline 36 & 0,206 & 0,091 & 1,000 & 0,201 & 0,187 & 1,000 & 0,695 & 27 & 1,043 \\
\hline 37 & 0,007 & 0,144 & 1,000 & 0,106 & 0,048 & 1,000 & 0,311 & 29 & 0,598 \\
\hline 38 & 0,492 & 0,192 & 1,000 & 0,473 & 0,889 & 1,000 & 0,770 & 21 & 0,821 \\
\hline 39 & 0,466 & 0,181 & 1,000 & 0,605 & 0,481 & 1,000 & 0,806 & 19 & 0,866 \\
\hline 40 & 0,999 & 0,959 & 1,000 & 0,998 & 1,000 & 0,999 & 0,990 & 45 & 1,752 \\
\hline 41 & 0,019 & 0,361 & 1,000 & 0,006 & 0,007 & 1,000 & 0,242 & 34 & $-0,070$ \\
\hline
\end{tabular}

Analisando a Tabela 2 verifica-se que dentre as distribuições analisadas apenas a distribuição Gama não se apresentou correspondente à distribuição dos dados em todos os casos testados pelo KS.

Dessa forma, os resultados obtidos diferem da afirmação de Naghettini \& Pinto (2007), os quais explicitam que a versatilidade de formas, o coeficiente de assimetria variável e positivo, aliados ao fato da variável aleatória não ser definida para valores negativos fazem da distribuição Gama um modelo probabilístico muito atraente para a representação de variáveis hidrológicas e hidrometeorológicas. Além disso, tal constatação descarta em parte a eficiência da distribuição 
Gama descrita por Haan (1977) na modelação de alturas de precipitação de durações diárias, semanais, mensais e anuais.

As análises realizadas, mostradas na Tabela 2, foram de séries temporais máximas anuais de um dia. Dessa maneira, os resultados apresentados anteriormente sobre a distribuição gama denotam que essa é mais adequada apenas para modelagem de períodos curtos de chuva, como conclui Murta et al. (2005), e se encontra evidenciado nos estudos de Moreira et al. (2010); Silva et al. (2007); e Vasconcellos et al. (1999).

A distribuição Exponencial assim como a distribuição Gama apresentaram um alto índice de rejeição quanto ao seu ajuste com os dados amostrais utilizados, sendo o seu uso recomendado apenas para 5 das 41 estações avaliadas. Tal fenômeno de reprovação também foi observado por Catalunha et al. (2002) ao testarem essa distribuição com dados pluviométricos do estado de Minas Gerais.

Os autores atribuíram a modelagem de tal elemento hidrológico à ineficiência da distribuição Exponencial. Esses autores ainda frisam sobre a superestimação que esse modelo estatístico provoca em grande parte dos dados de chuva. Desta forma, o KS demonstrou que os dados, em sua maioria, não possuem uma assimetria em forma de "J" invertido, característica típica da distribuição Exponencial que aprovaria o seu uso (CATALUNHA et al., 2002).

Torna-se possível constatar na Tabela 2 que não houve rejeição da aderência dos dados com as distribuições de Weibull, Log-Normal, Gumbel e Logística. Desta forma, o resultado da distribuição de Weibull concordou com a afirmação de Catalunha et al. (2002), o qual destaca o emprego desse modelo estatístico na análise hidrológica para eventos extremos, além de ressaltar a superioridade da mesma quando comparada com a distribuição Gama para a modelagem de dados de precipitação, como pode ser comprovado anteriormente.

Para os modelos estáticos de Log-Normal e Gumbel, o trabalho de Back (2001), confirma as constatações acima, pois tal autor ao selecionar uma distribuição estatística para descrever as chuvas extremas do estado de Santa Catarina entre os diversos modelos chegou a conclusão que a distribuição de Gumbel e Log-Normal apresentaram o melhor ajuste para a maioria das estações pluviométricas estudadas.

No que tange ao aceite para a distribuição Log-Normal, em todos os casos, ao contrário da distribuição Gama, revelou-se que as probabilidades dos dados pluviométricos analisados se encontram dispostos nos núcleos das curvas de distribuições e não em suas caldas, pois Tucci (2009) destaca que as caldas das distribuições Log-Normal e Gama, possuem similaridades, o que provocaria aceites de mesma natureza, dados a características assimétricas de tais modelos estatísticos.

Os resultados obtidos pela distribuição Logística mostraram que a mesma é passível de uso na modelagem de eventos pluviométricos extremos para os dados utilizados do estado de Rondônia, desta forma o KS atestou que tal distribuição pode ser aplicada em outra área, além das mencionadas no trabalho de Bittencourt (2001), como as áreas de saúde, economia, administração e educação.

Em análise a Tabela 2 se nota que houve um aceite de todos os dados para a distribuição Normal, mesmo os dados apresentando uma assimetria, característica essa que refutaria de forma automática a hipótese nula para essa distribuição empírica, visto que a disposição simétrica dos dados é um atributo latente a tal distribuição (NAGHETTINI \& PINTO, 2007).

Essa anormalidade pode ser explicada pela inferioridade do teste KS em confirmar a normalidade dos dados, sendo essa constatação encontrada no trabalho de Leotti et al. (2005), onde ao avaliarem dados que não continham normalidade, o teste Kolmogorov-Smirnov apresentou respostas não verídicas, afirmando que os mesmo eram normais, sendo que o KS, dentre todos os testes analisados, foi o que apresentou o pior desempenho quanto à avaliação de distribuição normal.

Os resultados da Tabela 2 revelam de forma geral que as respostas fornecidas pelo teste KS devem ser analisadas rigorosamente, pois o mesmo forneceu uma grande aceitação na maioria das distribuições, tal observação assemelha-se as constatações de Catalunha et al. (2002), o qual verificou que o nível de aprovação do teste Kolmogorov-Smirnov para diferentes distribuições de probabilidade é elevado, gerando dessa forma uma insegurança em seu uso.

$\mathrm{O}$ autor supracitado atribuiu tal fenômeno de aceitação elevada às condições assimétricas das distribuições estatísticas, pois essa característica confere valores mais elevados nas classes iniciais e menores nas finais, o que compromete o teste $\mathrm{KS}$ por esse medir as diferenças entre as probabilidades teóricas e empíricas.

Nesse sentido, Araújo et al. (2007) também verificaram essa alta abrangência de aceite do KS ao analisarem a aderência de dados pluviométricos, de algumas estação na bacia da Lagoa Mirim no Rio Grande do Sul, em relação as distribuições: Normal, Log-Normal, Log-Pearson III e Gumbel.

Porém, esses mesmos autores destacaram que ao realizarem a plotagem dos dados em papel de probabilidade apropriado, não houve aderência adequada dos dados observados aos do modelo teórico, ressaltando novamente que os resultados fornecidos pelo KS devem ser utilizados com parcimônia.

Porém, diante do exposto, cabe frisar que segundo Naghettini \& Pinto (2007) o teste de aderência é apenas uma das três ferramentas que deve ser levado em conta na seleção de uma distribuição probabilística empírica na representação de dados hidrológicos, sendo as outras duas: as características físicas do fenômeno em foco e as possíveis deduções teóricas quanto às propriedades distributivas da variável em questão.

Nesses termos, a literatura explicita que a distribuição de Gumbel torna-se a mais indicada na representação de eventos pluviométricos extremos, pois Hershfield \& Kohler (1960) ao analisarem os dados de milhares de estações pluviométricas nos Estados Unidos comprovaram que a distribuição de Gumbel se mostra mais eficiente em descrever o fenômeno das chuvas intensas.

Em tal contexto, os trabalhos de Beijo et al. (2003); Oliveira et al. (2000) e Silva et al. (2003), são referências no uso da distribuição de Gumbel para a modelagem de chuvas extremas, comprovando, desta forma, a aplicabilidade dessa distribuição em tal fenômeno.

\section{CONCLUSÃO}

O trabalho denotou que o teste de aderência KS de forma geral forneceu uma expressiva aceitação na maioria das distribuições estatísticas testadas para os dados pluviométricos. No entanto, quando se relaciona as 
características físicas dos fenômenos pluviométricos extremos e as deduções teóricas quanto às propriedades distributivas da mesma, vislumbrou-se que a distribuição de Gumbel é mais indicada na modelagem desses eventos.

\section{REFERÊNCIAS BIBLIOGRÁFICAS}

AGUIAR, R. G.; VON RANDOW, C.; PRIANTE FILHO, N.; MANZI, A. O.; AGUIAR, L. J. G.; CARDOSO, F. L. Fluxos de massa e energia em uma floresta tropical no sudoeste da Amazônia. Revista Brasileira de Meteorologia, São José dos Campos, v. 21, n., p. 248 257, 2006.

ALMEIDA, A. Q.; RIBEIRO, A.; PAIVA, Y. G.; RASCON, N. J. L.; LIMA, E. P. Geoestatística no estudo de modelagem temporal da precipitação. Revista Brasileira de Engenharia Agrícola e Ambiental, Campina Grande, v. 15, n. 4, p. 354-358, 2011.

ARANTES, E. J.; PASSIG, F. H.; CARVALHO, K. Q.; KREUTZ, C.; ARANTES, E. A. Análise das chuvas intensas da região noroeste do Paraná. OLAM - Ciência \& Tecnologia, Rio Claro, n. Esp., p. 31-47, 2009.

ARAÚJO, R. D.; DAMÉ, R. C. F.; TEIXEIRA, C. F. A.; ROSSKOFF, J. L.; TERRA, V. S. S.; GAYER, C. A. P.; FRAGA, D. S. Desempenho do teste KolmogorovSmirnov na seleção de modelos teóricos probabilísticos para predição da precipitação pluviométrica mensal provável na Bacia da Lagoa Mirim/RS. In: Encontro de Pós-Graduação, 9, 2007, Pelotas. Anais..., Pelotas: FAEM, 2007.

BACK, A. J. Seleção de distribuição de probabilidade para chuvas diárias extremas do estado de Santa Catarina. Revista Brasileira de Meteorologia, São José dos Campos, v. 16, n. 2, p. 211-222, 2001.

BEIJO, L. A.; MUNIZ, J.A.; VOLPE, C. A.; PEREIRA, G. T. Estudo da precipitação máxima em Jaboticabal (SP) pela distribuição de Gumbel utilizando dois métodos de estimação dos parâmetros. Revista Brasileira de Agrometeorologia, Sete Lagoas, v. 11, n. 1, p. 141-147, 2003.

BITTENCOURT, H. R. Comparação da discriminação logística com o método da máxima verossimilhança gaussiana na classificação de imagens digitais. Porto Alegre: UFGRS, 2001. 67 p.b Dissertação de mestrado.

BORGES, L. C.; FERREIR, D. F. Poder e taxas de erro tipo i dos testes Scott-Knott, Tukey e Student-Newman-Keuls sob distribuições normal e não normais dos resíduos. Revista de Matemática e Estatística, São Paulo, v. 21, n. 1, p. 67-83, 2003.

BRASIL. Departamento Nacional de Produção Mineral. Projeto RADAMBRASIL, Levantamento de Recursos Naturais. Folha SC - 20: Porto Velho. Rio de Janeiro, v. 16, 1978.
BUTT, N.; OLIVEIRA, P. A.; HEIL COSTA, M. H. Evidence that deforestation affects the onset of the rainy season in Rondônia, Brazil. Journal of Geophysical Research, v. 116, n. D11120, p. 1-8, 2011.

CATALUNHA, J. M.; SEDIYAMA, G. C.; RIBEIRO, A.; LEAL, B. G.; SOARES, C. P. B. Aplicação de cinco funções densidade de probabilidade a série de precipitação pluvial do estado de Minas Gerais. Revista Brasileira de Agrometeorologia, Sete Lagoas, v. 10, n. 1, p. 153-162, 2002

FIETZ, C. R.; COMUNELL, E. Probabilidade de ocorrência de chuva em Mato Grosso do Sul. Dourados: Embrapa Agropecuária Oeste, 2006. 184p.

HAAN, C. T. Statistical Methods in Hydrology. Ames (IA): The Iowa University Press, 1977. 378p.

HERSFIELD, D. M.; KOHLER, M. A. An empirical appraisal of the Gumbel extreme value procedure. Journal of Geophysical Research, v. 65, n. 6, p. 1737 1746, 1960.

IBGE. Instituto Brasileiro de Geografia e Estatística. Cidades por Unidades Federativas.: <http://www.ibge.gov.br/cidadesat/topwindow.htm?1>. 20 jun. 2011.

LEOTTI, V. B.; BIRCK, A. R.; RIBOLDI, J. Comparação dos Testes de Aderência à Normalidade KolmolgorovSmirnov, Anderson-Darling, Cramer-Von Mises e Shapiro-Wilk por simulação. In: Simpósio de Estatística Aplicada à Experimentação Agronômica, 11., 2005, Londrina. Anais..., Londrina: SEAEGRO, 2005. p. 192.

MURTA, R. M.; TEODORO, S. M.; BONOMO, P.; CHAVES, M. A. Precipitação pluvial mensal em níveis de probabilidade pela distribuição gama para duas localidades do sudoeste da Bahia. Ciência Agrotécnica, Lavras, v. 29, n. 5, p. 988-994, 2005.

NAGHETTINI, M.; PINTO, E. J. A. Hidrologia Estatística Belo horizonte: CRPM, 2007. 552 p.

OLIVEIRA, J. P. B.; CECÍLIO, R. C.; XAVIER, A. C.; JASPER, A. P. S.; OLIVEIRA, L. B. Precipitação provável para Alegre-ES através da distribuição de probabilidade gama. Engenharia Ambiental, Espírito Santo do Pinhal, v. 7, n. 2, p. 204-211, 2010.

OLIVEIRA, L. F. C.; VIOLA, M. R.; PEREIRA, S.; MORAIS, N. R. Modelos de predição de chuvas intensas para o estado do Mato Grosso, Brasil. Ambi-Agua, Taubaté, v. 6, n. 3, p. 274-290, 2011.

OLIVEIRA, L. F. C.; CORTÊS, F. C.; BARBOSA, F. O. A.; ROMÃO, P. A.; CARVALHO, D. F. Estimativa das equações de chuvas intensas para algumas localidades no estado de Goiás pelo método da desagregação de chuvas. Pesquisa Agropecuária Tropical, Goiânia, v. 30, n. 1, p. 23-27, 2000. 
RELIASOFT - ReliaSoft Corporation. Characteristics of the Weibull Distribution. Disponível em: <http://www.weibull.com/hotwire/issue14/relbasics14.ht $\mathrm{m}>.03$ dez. 2011.

RONDÔNIA. Secretaria de Estado do Desenvolvimento Ambiental (SEDAM). Boletim Climatológico de Rondônia - 2008. Porto Velho: SEDAM, 2009.

SILVA, D. D.; PEREIRA, S. B.; PRUSKI, F. F.; GOMES FILHO, R. R.; LANASE, A. M. Q.; BAENA, L. G. N. Equações de intensidade-duração-frequência da precipitação pluvial para o estado de Tocantins. Engenharia na Agricultura, Viçosa, v. 11, n. 1, p.7-14, 2003.

SILVA, J. C.; HELDWEIN, A. B.; MARTINS, F. B.; TRENTIN, G.; GRIMM, E. L. Análise de distribuição de chuva para Santa Maria, RS. Revista Brasileira de Engenharia Agrícola e Ambiental, Campina Grande, v. 11, n. 1, p. 67-72, 2007.

SOUZA, V. A. S. Eventos de precipitação extremas na Amazônia Ocidental: Rondônia - Brasil. Ji-Paraná: UNIR, 2012. 73p. Monografia Graduação.

TUCCI, C. E. M. (Org.). Hidrologia: Ciências e aplicação. 4. Ed. Porto Alegre: Ed. da Universidade: ABRH: EDUSP, 2009. 943 p.

TUCCI, C. E. M. Água no Meio Urbano. In: Aldo da Cunha Rebouças; Benedito Braga; José Galizia Tundisi. (Org.). Águas Doces no Brasil. 1 ed. São Paulo: Escrituras, 1999, cap. 12, p. 399-432.

TUCCI, C. E. M. Águas urbanas. Estudos Avançados, São Paulo, v. 22, n. 63, p. 97-112, 2008.

TUCCI, C. E. M. Inundações Urbanas. 1. Ed. Porto Alegre: ABRH, 2007.389 p.

VASCONCELLOS, S. L. B.; ANDRÉ, R. G. B.; PERECIN, D. Sequências de dias com e sem chuva no município de Jaboticabal-SP. Revista Brasileira de Meteorologia, São José dos Campos, v. 14, n. 2, p. 79-90, 1999.

WEBleR, A. D.; AGUiAR, R. G.; AGUiAR, L. J. G. Características da precipitação em área de floresta primária e área de pastagem no Estado de Rondônia. Revista Ciência e Natura, Santa Maria, v. Esp., p. 5558, 2007. 